\title{
Author Correction: Enantioselective hydrogenation of dialkyl ketones
}

Feng-Hua Zhang (D), Fa-Jian Zhang, Mao-Lin Li, Jian-Hua Xie (D) and Qi-Lin Zhou (1)

Correction to: Nature Catalysis https://doi.org/10.1038/s41929-020-0474-5, published online 22 June 2020.

In the version of this Article originally published, in Fig. $5 c$, an incorrect structure was shown for $(R)$-2-butanol. This has now been corrected in the online versions of this Article.

Published online: 4 August 2020

https://doi.org/10.1038/s41929-020-00502-z

๑) The Author(s), under exclusive licence to Springer Nature Limited 2020 